\title{
Rotating Ellis Wormholes in Four Dimensions
}

\author{
Burkhard Kleihaus ${ }^{1}$, Jutta Kunz ${ }^{1}$ \\ ${ }^{1}$ Institut für Physik, Universität Oldenburg \\ Postfach 2503, D-26111 Oldenburg, Germany
}

October 11, 2018

\begin{abstract}
We present rotating wormhole solutions in General Relativity, which are supported by a phantom scalar field. These solutions evolve from the static Ellis wormhole, when the throat is set into rotation. As the rotational velocity increases, the throat deforms until at a maximal value of the rotational velocity, an extremal Kerr solution is encountered. The rotating wormholes attain a finite mass and quadrupole moment. They exhibit ergospheres and possess bound orbits.
\end{abstract}




\section{Introduction}

Lorentzian wormholes are supposed to provide connections either between otherwise separate universes or within a single universe. These wormholes should not possess horizons, like the Einstein-Rosen bridge [1] or physical singularities. Instead they should be smooth solutions of the Einstein-matter equations with a nontrivial topology.

The simplest such wormhole is the Ellis wormhole [2, 3, 4]. Its throat is held open by the presence of a phantom field, i.e., a scalar field with a reversed sign in front of its kinetic term. In Einstein gravity this choice of sign is necessary in order to provide the required violation of the energy conditions. This was discussed in detail by Morris and Thorne [5, 6], whose motivation was to obtain traversable wormhole solutions in General Relativity. In recent years phantom fields have become ubiquitous in cosmology, providing one of the explanations for the observed accelerated expansion of the Universe [7].

While wormholes present a fascinating subject with their potential impact on space travel and the possibility of time travel [5, 8], they may also be viewed more mundane as potential astrophysical objects that may be searched for observationally [9, 10, 11]. In this respect, wormholes have for instance been considered as gravitational lenses, as first addressed in [12, 13, whose Einstein rings [14] or shadows [15, 16] have been studied. But also neutron star-wormhole systems and their possible astrophysical signatures have been addressed [18, 19, 20, 21].

Astrophysical objects generically carry spin. However, all of the smooth wormhole solutions of the Einstein-matter equations obtained so far (in four space-time dimensions), are either static or slowly rotating perturbative solutions [22, 23] $]$. Thus we here present for the first time a family of globally regular stationary wormhole solutions. These represent the rotating generalizations of the Ellis wormholes. We determine their domain of existence and analyze their physical properties. We note, that the family of rotating wormholes ends in an extremal Kerr black hole, and conclude, that it is a generic feature of families of wormholes in Einstein gravity to end in extremal black hole solutions.

\section{The model}

We consider Einstein gravity coupled to a phantom field $\Phi$ with action

$$
S=\int\left[\frac{c^{4}}{16 \pi G} R+\frac{\hbar^{2}}{2 m_{0}} \partial_{\mu} \Phi \partial^{\mu} \Phi\right] \sqrt{-g} d^{4} x,
$$

where $R$ denotes the curvature scalar, $G$ is Newton's constant, $g$ is the determinant of the metric, and $m_{0}$ represents a mass scale.

\footnotetext{
${ }^{1}$ Note, that the Teo wormhole $[24$ is not a solution of the Einstein-matter equations.
} 
To construct stationary rotating wormhole solutions of this action we employ the line element

$$
d s^{2}=-e^{f} c^{2} d t^{2}+p^{2} e^{-f}\left(e^{\nu}\left[d \eta^{2}+h d \theta^{2}\right]+h \sin ^{2} \theta(d \varphi-\omega d t)^{2}\right),
$$

where $f, p, \nu$ and $\omega$ are functions only of $\eta$ and $\theta$, and $h=\eta^{2}+\eta_{0}^{2}$ is an auxiliary function. We note, that the coordinate $\eta$ takes positive and negative values, i.e. $-\infty<$ $\eta<\infty$. The limits $\eta \rightarrow \pm \infty$ correspond to two distinct asymptotically flat regions. The phantom field $\Phi$ depends only on the coordinates $\eta$ and $\theta$, as well.

Substitution of these Ansätze into the Einstein equations leads to a system of nonlinear partial differential equations (PDEs). The PDE for the function $p$ decouples and has a simple form

$$
p_{, \eta, \eta}+\frac{3 \eta}{h} p_{, \eta}+\frac{2 \cos \theta}{h \sin \theta} p_{, \theta}+\frac{1}{h} p_{, \theta, \theta}=0 .
$$

A solution which satisfies the boundary conditions $p(\eta \rightarrow \infty)=p(\eta \rightarrow-\infty)=1$ and $p_{, \theta}(\theta=0)=p_{, \theta}(\theta=\pi)=0$ is given by $p=1$.

For the phantom field we then find with $p=1$ the PDE

$$
\Phi_{, \eta, \eta}+\frac{2 \eta}{h} \Phi_{, \eta}+\frac{\cos \theta}{h \sin \theta} \Phi_{, \theta}+\frac{1}{h} \Phi_{, \theta, \theta}=0 .
$$

An explicit solution is $\Phi_{, \eta}=D / h$, where the constant $D$ represents the scalar charge of the wormhole solution.

The remaining equations yield a set of 3 PDEs for the functions $f, \nu$ and $\omega$ together with an equation for the scalar charge and a constraint equation. We solve these PDEs numerically, while monitoring the fulfillment of the constraint and the constancy of the scalar charge.

We here focus on symmetric wormholes, where $f$ and $\nu$ are even functions of $\eta$. Their throat is located at the hypersurface $\eta=0$, which represents a minimal surface. The equatorial radius $R$ of the throat is given by

$$
R=\left.\sqrt{g_{\varphi \varphi}}\right|_{\eta=0, \theta=\pi / 2}=e^{-f_{0} / 2} \eta_{0}
$$

with $f_{0}=f(\eta=0, \theta=\pi / 2)$, while the polar radius $R_{p}$ and the areal radius $R_{A}$ are obtained via

$$
R_{p}=\left.\frac{\eta_{0}}{\pi} \int_{0}^{\pi} e^{(\nu-f) / 2}\right|_{\eta=0} d \theta, \quad R_{A}^{2}=\left.\frac{\eta_{0}^{2}}{2} \int_{0}^{\pi} e^{\nu / 2-f}\right|_{\eta=0} \sin \theta d \theta
$$

Denoting the angular velocity of the throat by $\Omega=\omega_{0}$, the dimensionless rotational velocity of the throat is given by

$$
v_{e}=\frac{R \Omega}{c} .
$$

The mass $M$ and angular momentum $J$ of the wormhole solutions are obtained from the asymptotic form of the metric tensor components

$$
g_{t t} \underset{\eta \rightarrow \infty}{\longrightarrow}-c^{2}\left(1-\frac{2 G M}{c^{2} \eta}\right), \quad g_{t \varphi} \underset{\eta \rightarrow \infty}{\longrightarrow}-\frac{2 G J}{c^{2}} \frac{\sin ^{2} \theta}{\eta}
$$


In order to establish a relation between the mass and the angular momentum, i.e., a Smarr-type relation, we consider the integrals

$$
\begin{aligned}
I_{M} & =\frac{c^{4}}{4 \pi G} \int_{\Sigma} R_{\mu \nu} n^{\mu} \xi^{\nu} d V=\left.\frac{1}{8 \pi G} \int\left[c^{2} h f_{, \eta} \sin \theta-h^{2} \omega \omega_{, \eta} e^{-2 f} \sin ^{3} \theta\right]\right|_{0} ^{\infty} d \theta d \varphi, \\
I_{J} & =-\frac{c^{4}}{8 \pi G} \int_{\Sigma} R_{\mu \nu} n^{\mu} \psi^{\nu} d V=\left.\frac{1}{16 \pi G} \int\left[c^{3} h^{2} \omega_{, \eta} e^{-2 f} \sin ^{3} \theta\right]\right|_{0} ^{\infty} d \theta d \varphi .
\end{aligned}
$$

Here $\Sigma$ is a spatial hypersurface extending from the throat $\eta=0$ to the asymptotically flat region $\eta \rightarrow \infty, d V$ denotes its natural volume element and $n^{\mu}$ is normal on $\Sigma$. $\xi^{\nu}$ is the time-like Killing vector field normalized to one in the asymptotically flat region $\eta \rightarrow \infty$, and $\psi^{\nu}$ is the space-like Killing vector field. In evaluating the expressions Eqs. 9.10, we have taken into account that the solutions are regular on the symmetry axis and possess reflection symmetry with respect to the equatorial plane. On the other hand the integrals have to vanish as a consequence of the Einstein equations. This yields for Eq. (10)

$$
\int c^{3}\left[h^{2} \omega_{, \eta} e^{-2 f}\right]_{\eta=0} \sin ^{3} \theta d \theta d \varphi=-16 \pi c G J,
$$

while substitution of this result in Eq. (9) leads to the desired mass relation

$$
M c^{2}=2 \omega_{0} J \quad \Longleftrightarrow \quad \frac{M}{M_{0}}=\frac{\Omega}{\Omega_{0}} \frac{J}{J_{0}}
$$

where $M_{0}=R c^{2} / G, \Omega_{0}=c / R$ and $J_{0}=R^{2} c^{3} / 2 G$.

To better understand the wormhole solutions we now turn to the discussion of the second asymptotic limit, $\eta \rightarrow-\infty$. As already noted for the perturbative solutions [22, 23], the function $\omega$ cannot tend to zero in both asymptotically flat regions, $\eta \rightarrow \infty$ and $\eta \rightarrow-\infty$. To see this in the general case, we express the PDE for $\omega$ in the form of a conservation law

$$
\left(h^{2} e^{-2 f} \sin ^{3} \theta \omega_{, \eta}\right)_{, \eta}+\left(h e^{-2 f} \sin ^{3} \theta \omega_{, \theta}\right)_{, \theta}=0
$$

and integrate over the full range of $\eta$ and $\theta$. Since $\omega_{, \theta}(0)=0=\omega_{, \theta}(\pi)$ we are left with

$$
\int_{0}^{\pi}\left[h^{2} e^{-2 f} \sin ^{3} \theta \omega_{, \eta}\right]_{\eta \rightarrow-\infty} d \theta=\int_{0}^{\pi}\left[h^{2} e^{-2 f} \sin ^{3} \theta \omega_{, \eta}\right]_{\eta \rightarrow \infty} d \theta \propto J .
$$

Hence, both limits are regular and non-vanishing for rotating wormholes. Next we multiply Eq. (13) by $\omega$ and integrate again. This yields

$$
\int_{0}^{\pi}\left[h^{2} e^{-2 f} \sin ^{3} \theta \omega \omega_{, \eta}\right]_{\eta \rightarrow-\infty}^{\eta \rightarrow \infty} d \theta=\int\left[h^{2} e^{-2 f} \sin ^{3} \theta\left(\omega_{, \eta}^{2}+\frac{1}{h} \omega_{, \theta}^{2}\right)\right] d \eta d \theta>0 .
$$

Since the term $h^{2} e^{-2 f} \omega_{, \eta}$ is bounded (in magnitude) from above in both limits, the function $\omega$ has to be non-zero in (at least) one of the asymptotic regions. We note, that one can always arrange $\omega(\infty)=0$ by adding a suitable constant. In this case $\omega(-\infty)=2 \omega_{0}$. Still, the region $\eta \rightarrow-\infty$ is asymptotically flat, as one can see by means of a simple coordinate transformation. In that case, $\omega(\infty) \rightarrow-2 \omega_{0}$. 


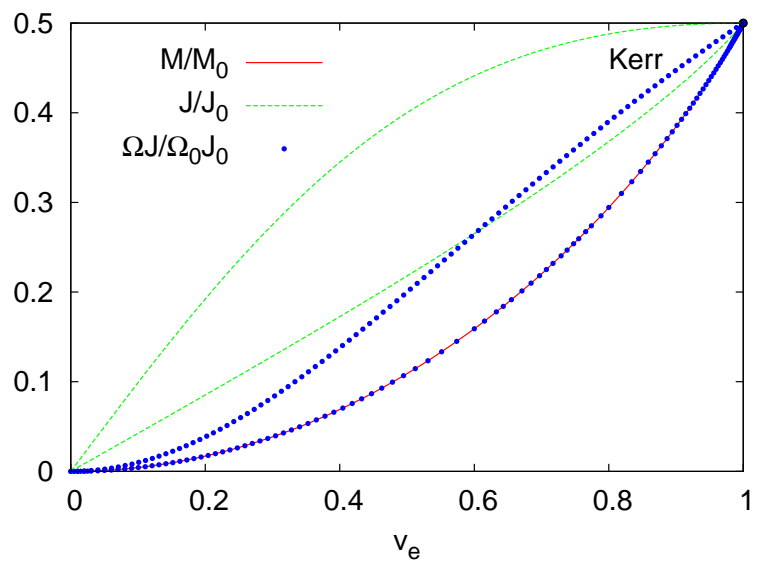

(a)

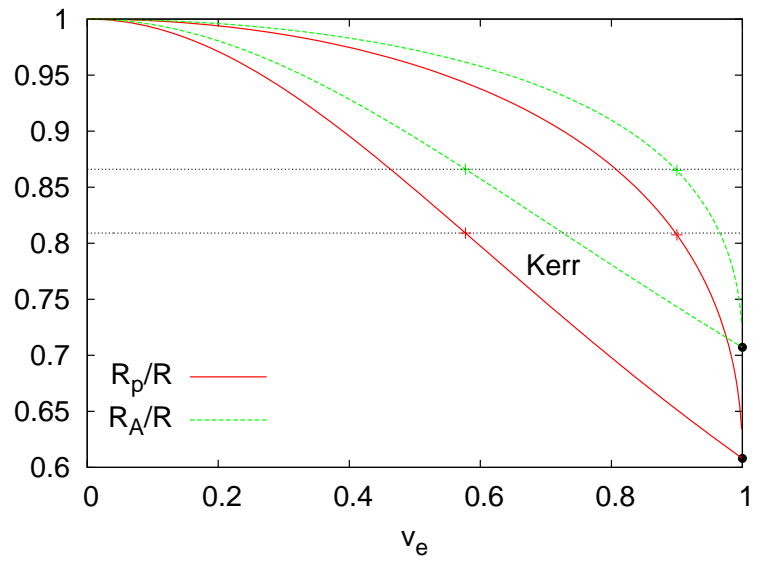

(b)

Figure 1: (a) The dimensionless mass $M / M_{0}$ and angular momentum $J / J_{0}$ versus the dimensionless rotational velocity $v_{e}$ for symmetric wormhole solutions. Validity of the Smarr-type relation Eq. (12) is also demonstrated for the wormhole solutions. (b) The polar radius $R_{p} / R$ and areal radius $R_{A} / R$ for fixed equatorial radius $R$ versus $v_{e}$. The crosses mark the onset of a negative Gaussian curvature at the poles. The black dots at $v_{e}=1$ in (a) and (b) correspond to the values of the extremal Kerr black hole. For comparison the respective curves for the full set of Kerr solutions are also shown (note that $M / M_{0}=1 / 2$ in (a)).

\section{Numerical results}

We exhibit in Fig. 1(a) the dimensionless mass and angular momentum of the family of symmetric rotating wormholes, evolving from the static Ellis wormhole. As the dimensionless rotational velocity $v_{e}$ reaches its maximal value, $v_{e}=1$, the hypersurface $\eta=0$ changes its character and a degenerate horizon forms. The limiting configuration represents an extremal Kerr black hole.

The scalar charge on the other hand is a monotonically decreasing function of $v_{e}$, which vanishes in the limit $v_{e}=1$, as it should for a Kerr black hole. Indeed, the phantom field vanishes identically in the limit. Accordingly, the Smarr-type relation (12) holds for Kerr black holes in the extremal limit, when $\omega_{0}$ denotes the horizon angular velocity.

When the spherically symmetric Ellis wormhole is set into rotation, the throat deforms. In particular, as seen in Fig. 1(b), the ratio of the polar radius to the equatorial radius $R_{p} / R$ decreases monotonically and assumes the value of the ratio of the corresponding extremal Kerr horizon radii in the limit $v_{e} \rightarrow 1$. The same holds for the areal radius $R_{A}$. Beyond a certain value of $v_{e}$ the Gaussian curvature turns negative at the poles of the throat, as indicated by the crosses in the figure. Interestingly, this occurs for the same value of the ratio $R_{p} / R$ for the wormholes as for the Kerr black holes, and 


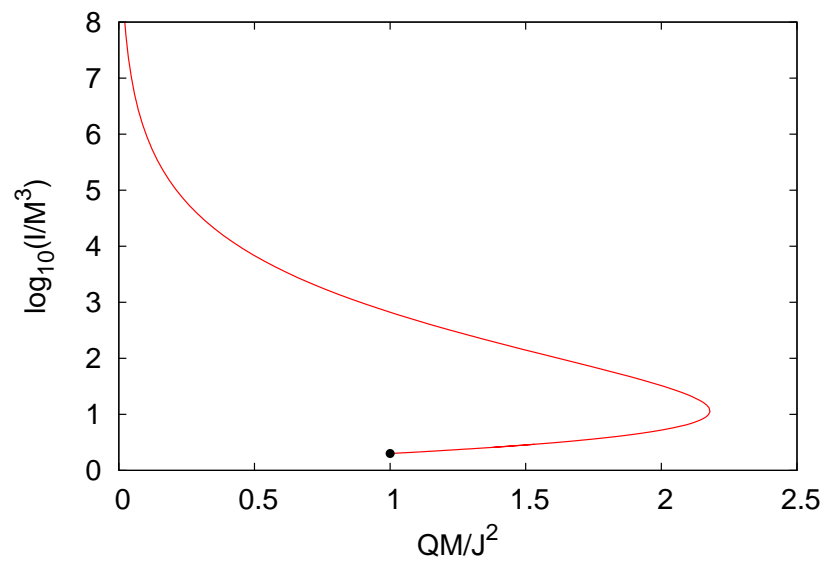

(a)

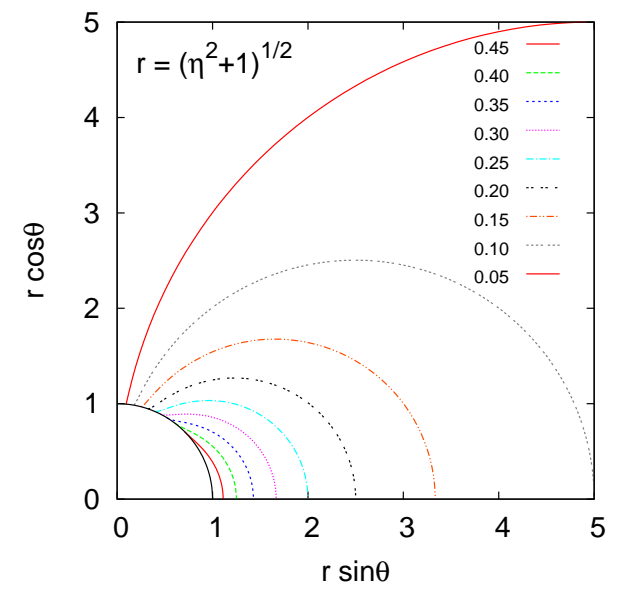

(b)

Figure 2: (a) The dimensionless moment of inertia $I / M^{3}$ versus the dimensionless quadrupole moment $Q M / J^{2}$. The black dot at $\nu_{e}=1$ corresponds to the value of the extremal Kerr black hole. (b) Quadrant of the ergoregion and the throat in coordinates $r$ and $\theta$ for fixed $\varphi, \eta_{0}=1$ and several values of $\omega_{0}$.

likewise for the ratio $R_{A} / R$.

Following [23] we consider the violation of the null energy condition (NEC) by evaluating the quantity $\Xi=R_{\mu \nu} k^{\mu} k^{\nu}$ with null vector $k^{\mu}=\left(e^{-f / 2}, e^{f / 2-\nu / 2}, 0, \omega e^{-f / 2}\right)$. Since $\Xi$ is non-positive the NEC is violated everywhere. Introducing a global scale invariant measure for the NEC violation, $Y=\frac{1}{R} \int \Xi \sqrt{-g} d \eta d \theta d \varphi$, we observe a monotonic decrease of $|Y|$ with increasing rotation velocity $v_{e}$.

To extract the quadrupole moment $Q$ of the rotating wormhole solutions we follow Geroch and Hansen [25, 26] and extract it from the asymptotic expansion in appropriate coordinates (see e.g. [27]). We recall, that the dimensionless quadrupole moment $Q M / J^{2}$ of the Kerr solutions is constant, $Q M / J^{2}=1$. The dimensionless quadrupole moment $Q M / J^{2}$ of the family of rotation wormhole solutions is shown in Fig. 2(a), It is smaller than the Kerr value for slow rotation while it exceeds the Kerr value for fast rotation. In the limit $v_{e} \rightarrow 1$, however, the Kerr value is attained. The dimensionless moment of inertia of the Kerr solutions is bounded and given by $I / M_{\text {Kerr }}^{3}=2\left(1+\sqrt{1-J^{2} / M^{4}}\right)$. For rotating wormholes, however, $I / M^{3}$ rises without bound in the limit of slow rotation, since the static Ellis wormhole has $M=0$.

Rotating wormholes possess an ergoregion, defined by the condition $g_{t t}>0$,

$$
g_{t t}=-e^{f} c^{2}+e^{-f} h \sin ^{2} \theta \omega^{2}>0 .
$$

While the rotation axis is excluded from the ergoregion, its boundary in the equatorial plane is closely related to the angular velocity $\omega_{0}$ of the throat. The numerical calculations yield with high accuracy the relation $\arctan \left(\eta_{\text {ergo }} / \eta_{0}\right)=\arccos \left(2 \omega_{0}\right)$. Thus an ergoregion exists in the asymptotically flat region, where $\omega \rightarrow 0$, only for sufficiently 


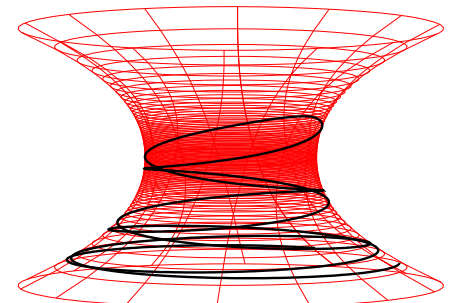

Figure 3: Embedding diagram of the bound orbit of a test particle in the equatorial plane of a rotating wormhole $\left(v_{e}=0.966\right)$.

fast rotation, while there always exists an ergoregion in the asymptotically flat region, where $\omega$ tends to a finite value. The ergoregion is exhibited in Fig. 2(b).

In contrast to the static Ellis wormhole, rotating wormholes possess stable bound orbits. Indeed, there are two types of bound orbits, those that always remain within a single universe, and those that oscillate between the two universes. An example of such a two-world bound orbit is seen in Fig. 3. For corotating orbits the innermost stable circular orbit (ISCO) resides at the throat. For counterrotating orbits, however, the radius of the ISCO depends on the rotation velocity, with the ratio $R_{\mathrm{ISCO}} / R$ increasing almost by a factor of two from the static limit to the extremal Kerr black hole. At the same time the ratio of the orbital period $T_{\mathrm{ISCO}} / T$, where $T$ denotes the orbital period of the throat (or the horizon of the extremal black hole), increases roughly by a factor of three.

\section{Conclusions}

While wormholes with phantom fields have been studied in numerous respects (see e.g. [6]), we have here provided the first globally regular rotating wormhole solutions and analyzed their physical properties, such as their global charges including their quadrupole moments and moments of inertia, as well as the properties of their throats. In particular, we obtained a Smarr-type relation for these wormholes.

With increasing rotation velocity the violation of the NEC decreases. The family of rotating wormholes then ends in an extremal Kerr black hole. We note, that it seems to be a generic feature, that the families of generalized Ellis wormholes end in an extremal black hole. In particular, charged static Ellis wormholes in 4 dimensions end in an extremal Reissner-Nordström black hole [28], while rotating Ellis wormholes in 5 dimensions end in an extremal Myers-Perry black hole [29]. 
While the solutions presented are symmetric, in general rotating wormholes need not be symmetric. By choosing non-symmetric boundary conditions for the metric function $f$, families of rotating wormholes emerge, which have different masses associated to their two asymptotically flat regions, as manifest already in the static case. As the asymmetry increases, the wormhole properties change. But no matter how asymmetric the solutions are, these families always appear to end in an extremal Kerr black hole.

Concerning the stability of the wormhole solutions, it is known that the static Ellis wormholes are unstable [30, 31, 32]. However, it has been argued, that rotating wormholes might be stable [33]. Indeed, a stability analysis of five-dimensional rotating wormholes has shown, that the unstable mode of the static solutions disappears, when the rotation is sufficiently fast [29]. Whether this will happen as well in four dimensions still needs to be shown. On the other hand, stability might also be achieved by allowing higher curvature terms in the action [34, 35].

\section{Acknowledgment}

We gratefully acknowledge support by the DFG Research Training Group 1620 "Models of Gravity".

\section{References}

[1] A. Einstein, N. Rosen, Phys. Rev. 48 (1935) 73.

[2] H. G. Ellis, J. Math. Phys. 14, 104-118 (1973).

[3] H. G. Ellis, Gen. Rel. Grav. 10, 105-123 (1979).

[4] K. A. Bronnikov, Acta Phys. Polon. B4, 251-266 (1973).

[5] M. S. Morris, K. S. Thorne, Am. J. Phys. 56, 395-412 (1988).

[6] M. Visser, "Lorentzian wormholes: From Einstein to Hawking." - Woodbury, New York, 1996. - 412 p.

[7] F. S. N. Lobo, Phys. Rev. D 71, 084011 (2005).

[8] M. S. Morris, K. S. Thorne and U. Yurtsever, Phys. Rev. Lett. 61, 1446 (1988).

[9] F. Abe, Astrophys. J. 725, 787 (2010).

[10] Y. Toki, T. Kitamura, H. Asada and F. Abe, Astrophys. J. 740, 121 (2011).

[11] R. Takahashi and H. Asada, Astrophys. J. 768, L16 (2013).

[12] J. G. Cramer, R. L. Forward, M. S. Morris, M. Visser, G. Benford and G. A. Landis, Phys. Rev. D 51, 3117 (1995).

[13] V. Perlick, Phys. Rev. D 69, 064017 (2004). 
[14] N. Tsukamoto, T. Harada and K. Yajima, Phys. Rev. D 86, 104062 (2012).

[15] C. Bambi, Phys. Rev. D 87, 107501 (2013).

[16] P. G. Nedkova, V. K. Tinchev and S. S. Yazadjiev, Phys. Rev. D 88, no. 12, 124019 (2013).

[17] C. Armendariz-Picon, Phys. Rev. D65, 104010 (2002).

[18] V. Dzhunushaliev, V. Folomeev, B. Kleihaus and J. Kunz, JCAP 1104, 031 (2011).

[19] V. Dzhunushaliev, V. Folomeev, B. Kleihaus and J. Kunz, Phys. Rev. D 85, 124028 (2012).

[20] V. Dzhunushaliev, V. Folomeev, B. Kleihaus and J. Kunz, Phys. Rev. D 87, 104036 (2013).

[21] V. Dzhunushaliev, V. Folomeev, B. Kleihaus and J. Kunz, Phys. Rev. D 89, 084018 (2014).

[22] P. E. Kashargin and S. V. Sushkov, Grav. Cosmol. 14, 80 (2008).

[23] P. E. Kashargin and S. V. Sushkov, Phys. Rev. D 78, 064071 (2008).

[24] E. Teo, Phys. Rev. D 58, 024014 (1998).

[25] R. P. Geroch, J. Math. Phys. 11, 2580 (1970).

[26] R. O. Hansen, J. Math. Phys. 15, 46 (1974).

[27] B. Kleihaus, J. Kunz and S. Mojica, Phys. Rev. D, in press, arXiv:1407.6884 [gr-qc].

[28] O. Hauser, R. Ibadov, B. Kleihaus and J. Kunz, Phys. Rev. D 89, 064010 (2014).

[29] V. Dzhunushaliev, V. Folomeev, B. Kleihaus, J. Kunz and E. Radu, Phys. Rev. D 88, 124028 (2013).

[30] H. -a. Shinkai and S. A. Hayward, Phys. Rev. D 66, 044005 (2002).

[31] J. A. Gonzalez, F. S. Guzman, and O. Sarbach, Class. Quant. Grav. 26, 015010 (2009).

[32] J. A. Gonzalez, F. S. Guzman, and O. Sarbach, Class. Quant. Grav. 26, 015011 (2009).

[33] T. Matos and D. Nunez, Class. Quant. Grav. 23, 4485 (2006).

[34] P. Kanti, B. Kleihaus and J. Kunz, Phys. Rev. Lett. 107, 271101 (2011).

[35] P. Kanti, B. Kleihaus and J. Kunz, Phys. Rev. D 85 (2012) 044007. 